\title{
PARTICIPACIÓN DE LA MUJER EN BOYACÁ EN LOS CONSEJOS MUNICIPALES DE JUVENTUD Y LOS ORGANISMOS COMUNALES PARA 2011
}

\author{
Doris Amalia Alba Sánchez ${ }^{1}$
}

Recibido: 28 de mayo de 2013 Aceptado: 12 de agosto de 2013

\section{Resumen}

La participación de la mujer cuenta con significativos avances en Colombia, especialmente en la normatividad. Se resalta la expedición de la ley Estatutaria 581 de 2000, o Ley de cuotas, la Ley Estatutaria 1475 de 2011, la Ley 1434 de 2011 que crea la Comisión Legal para la equidad de la Mujer en el Congreso, la Ley 1450 de 2011 que crea la Alta Consejería para la Equidad de la Mujer y el CONPES 140 de 2011 que genera lineamientos para el logro de los ODM en 2015. Teniendo como referente este marco normativo y una investigación de tipo descriptivo, realizada por el DAP de Boyacá, cuyo objetivo consistió en analizar de manera cuantitativa la participación de la mujer en algunos escenarios de gestión social, que se constituyen en dos importantes semilleros de liderazgo en Boyacá para 2011: los Consejos Municipales de juventud y los organismos comunales en donde la mayor participación de la mujer por órganos se da en el de conciliación con un 42\%, seguida del de Administración con un $35 \%$, del de Ejecución con un 31\%. El de Representación alcanza un $27 \%$ y el de control un $24 \%$. Se generan análisis y nuevas preguntas de investigación que conduzcan a la Academia y el Estado a ofrecer las garantías para la participación de la mujer, y a reflexionar frente a la pertinencia del derecho adquirido y las condiciones para ejercerlo.

Palabras claves: Equidad de género, participación de la mujer, organismos comunales, ODM 3

1 Economista, Especialista en Planeación y Gestión del Desarrollo Territorial, Magister en Desarrollo

Educativo y Social, Asesor parlamentario, Asesor de Política Social, Profesional Línea de Infancia,

Familia y Mujer Departamento Administrativo de Planeación de Boyacá, Docente Universitario y

SENA, actualmente Docente Ocasional UNAD 


\section{PARTICIPATION OF THE WOMAN IN BOYACÁ IN THE MUNICIPAL BOARD OF YOUTH AND THE COMMUNAL ORGANISMS FOR 2011}

\section{Abstract}

The participation of the woman possesses significant advances in Colombia, specially in the local laws. The expedition of the Statutory law 581 of 2000 is highlighted, or Law of quotas, the Statutory Law 1475 of 2011, the Law 1434 of 2011 creates the Legal Commission for the equity of the Woman in the Congress, the Law 1450 of 2011 creates the High Council for the Equity of the Woman and the CONPES 140 of 2011 that generates guidelines for the achievement of the ODM in 2015. Taking this normative frame and descriptive research, conducted by the DAP of Boyacá, whose purpose was to quantitatively analyze the participation of women in social management scenarios will be reviewed as a modal two important seedbeds of leadership are analyzed in Boyacá for 2011: the Municipal board of youth and the communal organisms where the major participation of the woman by organs is met in that of conciliation by $42 \%$, followed by that of Administration board by $35 \%$, of that of Execution by $31 \%$. The Representation board reaches $27 \%$ and the Control board $24 \%$. The analysis generates new questions of investigation that will lead the Academy and the State to offer the guarantees for the participation of the woman, and to think about the relevancy of the acquired right and the conditions to exercise it.

Keywords: Gender equality, Participation of the woman, Communal organisms, ODM 3

\section{Introducción}

Hablar de participación genera un compromiso muy importante y más cuando se trata de géne$\mathrm{ro}^{2}$. En las líneas que siguen se pretende realizar una reflexión frente a la participación de la mujer en Boyacá en dos escenarios que a juicio de la autora se convierten en semilleros de liderazgo y participación efectiva: los Consejos municipales de Juventud y los organismos comunales.

Se inicia la reflexión realizando una somera presentación del marco normativo en materia de participación de la mujer en Colombia, para ofrecer un contexto que ubique la discusión planteada.

El Fondo para el Logro de los ODM 2010, define Género como lo que «alude al distinto significado social que tiene el hecho de ser mujer y hombre, es decir, es una definición específica cultural de la feminidad y la masculinidad que varía en el tiempo y en el espacio. Este marco sitúa las relaciones de las mujeres y hombres en "contexto", permitiendo enfocarse en los procesos y relaciones que reproducen y refuerzan las desigualdades entre ambos, visibilizando la cuestión del poder que subyace en las relaciones de género»(P. 67)
Posteriormente, se presenta una revisión de algunos de los resultados de una investigación de tipo descriptivo ${ }^{3}$, realizada por el Departamento Administrativo de Planeación de Boyacá , cuyo objetivo consistió en analizar de manera cuantitativa la participación de la mujer en algunos escenarios de gestión social, utilizando el método inductivo - definido por Méndez 1998 como un «proceso de conocimiento que se inicia por la observación de fenómenos particulares con el propósito de llegar a conclusiones y premisas generales que pueden ser aplicadas a situaciones similares a la observada» (p. 131) - con la pretensión de realizar una medición del número y porcentaje de mujeres que participa-

\footnotetext{
3 Tamayo 2002 define los alcances de la investigación descriptiva así: "comprende la descripción, registro, análisis e interpretación de la naturaleza actual, y la composición o procesos de los fenómenos. El enfoque se hace sobre conclusiones dominantes 0 sobre cómo una persona, grupo o cosas se conduce o funciona en el presente» ( $p$ 46).
} 
ron en los Consejos municipales de juventud y los organismos comunales en el año 2011, y reflexionar sobre los resultados obtenidos y las posibles causas que los generaron, así como los lugares de mayor ocurrencia de los eventos.

Se presentan datos departamentales y municipales y las conclusiones más importantes que generan recomendaciones en materia de política pública. Para adelantar la investigación por parte del DAP Boyacá se acudió a fuentes primarias, mediante el procesamiento de las bases de datos de los Organismos Comunales de Boyacá, y los resultados del proceso electoral de los Consejos Municipales de Juventud adelantados hasta 2011, estadísticas suministradas por la Secretaría de Participación y Democracia, de la Gobernación de Boyacá.

Claramente la reflexión se debate en un enfoque con perspectiva de género. El Fondo para el logro de los ODM 2010 precisa «El enfoque de género para el PNUD supone una forma de observar la realidad que implica una mirada mas profunda que permite identificar los diferentes papeles y tareas que llevan a cabo los hombres y las mujeres en una sociedad, tanto las asimetrías como las relaciones de poder e inequidades. Ayuda a reconocer las causas que las producen y a formular mecanismos para superar las brechas. Contribuye a explicar y a ampliar aspectos de la realidad que anteriormente no habían sido tomados en cuenta, y es aplicable a todos los ámbitos de la vida: laboral, educativa, personal, etc.» (P. 67), por ello, es importante revisar el comportamiento de la participación bajo el criterio de género en el Departamento de Boyacá.

Como lo afirma Barraza (2007) «Aumentar la gobernabilidad democrática incorporando los derechos de las mujeres y el enfoque de género implica la construcción de políticas públicas que respondan a las demandas de los distintos gru-

4 DAP : Departamento Administrativo de Planeación pos de mujeres que integran la sociedad, brindándoles condiciones de seguridad y bienestar, a la vez que se fortalecen las expresiones de participación para las mujeres y su vinculación a los puestos de toma de decisión.

Las políticas públicas dirigidas a las mujeres son hoy necesarias básicamente porque permiten impulsar acciones que garanticen el desarrollo, al reconocerle a las mujeres un papel clave como agentes de desarrollo, y a la vez porque son políticas que permiten hacer efectivo el reconocimiento de los derechos de las mujeres y, por tanto, le reconocen su actoría social y política como ciudadanas. De este modo articulan a la lógica del desarrollo el reconocimiento de derechos, permitiendo que las políticas públicas vayan más allá de la superación de la pobreza, al hacer real la igualdad de oportunidades para todas las personas que componen la sociedad» (P.11).

Tradicionalmente los hombres han ocupado los cargos de representación social y política y por ello resulta relevante analizar como se viene gestando la participación de la mujer en escenarios que le son próximos y que le permiten tramitar por los caminos de la representación social y política, el primero en la etapa de la juventud, que representa los inicios de la participación crítica y devela los intereses que con el tiempo se fortalecerán como profesiones u ocupaciones y la segunda : la de las organizaciones comunales que van preparando a quienes las integran en el oficio, siempre interesante, del servicio comunitario, la solución de los problemas que permiten mejorar el día a día de los ciudadanos y que transforman las realidades locales, a partir del compromiso que se le imprime a la gestión allí realizada.

En escenarios como estos nace la afinidad con la representación social y se va adquiriendo la experticia en el manejo y trámite de los problemas sociales. Por ello es fundamental revisar el grado de participación de la mujer, 
en esos niveles de organización y representación, en un siglo que promueve y espera una participación activa y decidida de la mujer, imprimiendo un tinte más social en la toma de decisiones públicas.

\section{Antecedentes y marco normativo}

La participación de la mujer ha sido uno de los intereses fundamentales relacionados con la equidad de género en el mundo entero. Se ha llegado a acuerdos internacionales ${ }^{5}$ para gestionar el proceso de cambio y permitir que su voz llegue a los diferentes escenarios de la vida pública ${ }^{6}$. Muestra de ello es el compromiso emitido frente a los objetivos de desarrollo del milenio, cuyo objetivo Nro. 3 es Promover la igualdad entre los géneros y la autonomía de la mujer, encargo que tiene metas claras con medición para 2015.

La meta Universal resaltada por PNUD 2012 es «Eliminar las desigualdades entre ambos sexos en la enseñanza primaria y secundaria, preferiblemente para el año 2005 y en todos los niveles de enseñanza para 2015». Colombia se comprometió como meta a «Lograr la igualdad de género y la autonomía de la mujer» y en materia de participación «Incrementar por encima del $30 \%$ la participación de la mujer en los niveles decisorios de las diferentes ramas y órganos del poder público» ( $p 50)$.

\footnotetext{
Como lo consigna PNUD, 2004 en el Informe sobre Desarrollo Humano 2003, «En septiembre de 2000, los líderes mundiales reunidos en la Cumbre del Milenio de las Naciones Unidas se comprometieron a redoblar sus esfuerzos en favor de la paz, los derechos humanos, la democracia, la gobernabilidad, la sostenibilidad ambiental y la erradicación de la pobreza, y a promover los principios de la dignidad humana, la igualdad y la equidad. El documento resultante, la Declaración del Milenio, que fue aprobado por 189 países, incluye compromisos colectivos urgentes para eliminar la pobreza que aún padece una parte importante de la población mundial. Esta vez los líderes del mundo no se conformaron con que todo siguiera su curso, porque eran conscientes de que eso no era suficiente, sino que se comprometieron a cumplir unos objetivos ambiciosos dentro de unos plazos claramente establecidos. En la cumbre de 2000, la Asamblea General de las Naciones Unidas pidió también a su Secretario General que preparara una guía para conseguir los compromisos adquiridos en la Declaración: que los Objetivos de Desarrollo del Milenio, un documento con 8 objetivos, 18 metas específicas y 48 indicadores» (P. 28)

En Fondo para el logro de los ODM 2010, se hace una revisión de estos convenios internacionales frente a los derechos de la mujer, aquí se enfatiza el tema de participación: Convención sobre los derechos políticos de la Mujer ONU - 1952, Nueva York; Convención Internacional sobre la eliminación de todas las formas de Discriminación Racial - ONU, 1965; Conferencia Mundial sobre la mujer: «Declaración de México sobre la igualdad de la mujer y su contribución al Desarrollo y a paz», 1975; ...; Convención para la eliminación de Todas las Formas de Discriminación contra la mujer, CEDAW ONU, 1979;...; Conferencia Mundial de Derechos Humanos: «Declaración de Viena", enfatiza derechos de las mujeres y las niñas. Viena 1993 Cumbre Mundial sobre Desarrollo Social. Abordó temas de género y la necesidad de participación de las mujeres en el desarrollo socio - económico. Igualdad y equidad entre hombres y mujeres. Copenhague, 1995. p 70-71
}

Se requiere dejar atrás el estigma de la desigualdad de la mujer, como lo señala Sánchez 2012 «Hasta 1957, el proceso electoral podría decirse que era excluyente. En ese entonces, la sociedad colombiana no concebía la igualdad de género y solo una parte de la población ejercía algunos derechos enmarcados en un concepto de libertad diferente al generalizado y vigente hoy. La idea de incluir a las mujeres en la actividad electoral se contempló en 1954, se consolidó en las urnas el 1 de Diciembre de 1957 y forma parte del largo e inacabado camino de reivindicación de los Derechos Humanos... el 25 de Agosto de 1954 se promulgó el Acto Legislativo Nro. 3 y se aprobó una ley que permitía a las mujeres votar» (p. 1)Desde ese entonces, en Colombia se ha avanzado significativamente en el proceso de participación de la mujer en los escenarios de decisión, por lo menos en la parte normativa ${ }^{7}$. A continuación se presentará la normatividad mas reciente y que a juicio de la autora ilustra mejor la reflexión.

La conocida popularmente como Ley de cuotas regula en su Artículo 4. Colombia. Diario Oficial . Ley estatutaria por la cual se reglamenta la adecuada y efectiva participación de la mujer en los niveles decisorios de las diferentes ramas y órganos del poder público, de conformidad con los artículos 13, 40 y 43 de la Constitución Nacional y se dictan otras disposiciones. 31 de mayo de 2000. L estatutaria 581/2000, 1-2. «Participación efectiva de la mujer, considera: La participación adecuada de la mujer en los niveles del poder público definidos en los artículos 2 y 3 de la presente ley, se hará efectiva aplicando por parte de las autoridades nominadoras las siguientes reglas: a) Mínimo el treinta por ciento $(30 \%)$ de los cargos de máximo nivel decisorio,

\footnotetext{
En Fondo para el logro de los ODM 2010, se hace una presentación de la normatividad Colombiana destacándose para el tema que nos ocupa: «Ley 22 de 1981: Eliminación de todas las formas de discriminación racial; ...; Ley 51 de 1981: Eliminación de todas las formas de discriminación contra la mujer;...; Ley 581 del 2000: Reglamenta la adecuada y efectiva participación de la mujer en los niveles decisorios de las diferentes ramas y órganos del poder público; ...; Documento CONPES Social 91 de 2005: Metas y estrategias del milenio para Colombia 2015; Ley 823 de 2003: Consagra normas para la igualdad de oportunidades de las mujeres...,» (p. 73).
} 
de que trata el artículo 2 , serán desempeñados por mujeres; b) Mínimo el treinta por ciento (30\%) de los cargos de otros niveles decisorios, de que trata el artículo 3, serán desempeñados por mujeres. Parágrafo. El incumplimiento de lo ordenado en este artículo constituye causal de mala conducta, que será sancionada con suspensión hasta de treinta (30) días en el ejercicio del cargo, y con la destitución del mismo en caso de persistir en la conducta, de conformidad con el régimen disciplinario vigente».

Mediante Decreto 3545 de 2010 se crea la Alta Consejería Presidencial para la Equidad de la Mujer. En Colombia. Presidencia de la República. Decreto por el cual se crean unas Altas Consejerías en el Departamento Administrativo de la Presidencia de la República. 17 de septiembre de 2010. D 3445/2010, 7. « Artículo 20. La Alta Conserjería Presidencial para la Equidad de la Mujer, tendrá las siguientes funciones: 1 . Asistir al Presidente y al Gobierno Nacional en el diseño de las políticas gubernamentales destinadas a promover la equidad entre mujeres y hombres, siguiendo las orientaciones generales trazadas por el Presidente de la República.;....; 4. Establecer alianzas estratégicas con el sector privado, organismos internacionales, ONG, universidades y centros de investigación, para estimular y fortalecer la investigación y el análisis del conocimiento existente sobre la condición y situación de la mujer. 5. Apoyar organizaciones solidarias, comunitarias y sociales de mujeres a nivel nacional y velar por su participación activa en las acciones y programas estatales».

Posteriormente se genera el espacio en el legislativo, como puede leerse en Colombia. Diario Oficial. Por la cual se modifica y adiciona la Ley 5 de 1992, se crea la Comisión Legal para la Equidad de la Mujer del Congreso de la República de Colombia y se dictan otras disposiciones. 6 de Enero de 2011, L 1434/2011, 1. «Artículo 1. Objeto. La presente ley tiene por objeto fomentar la participación de la mujer en el ejercicio de la labor legislativa y de control político a través de la creación de la Comisión Legal para la Equidad de la Mujer del Congreso de la República».

Se extiende la regulación en cuanto a la participación con mayor equidad de género a los partidos y movimientos políticos. En Colombia. Diario Oficial . Ley estatutaria por medio de la cual se adoptan Reglas de organización y funcionamiento de los partidos y movimientos políticos, de los procesos electorales y se dictan otras disposiciones. 14 de Julio de 2011, L estatutaria 1475/2011, 12. En el «Título III De las Campañas Electorales, Capitulo I que trata de la inscripción de candidatos considera en el Articulo 28. Los partidos y movimientos políticos con personería jurídica podrán inscribir candidatos a cargos y corporaciones de elección popular previa verificación del cumplimiento de las calidades y requisitos de sus candidatos, así como de que no se encuentran incursos en causales de inhabilidad o incompatibilidad. Dichos candidatos deberán ser escogidos mediante procedimientos democráticos, de conformidad con sus estatutos. Las listas donde se elijan 5 o más curules para corporaciones de elección popular o las que se sometan a consulta -exceptuando su resultado- deberán conformarse por mínimo un 30\% de uno de los géneros».

Con la expedición del Plan Nacional de Desarrollo Prosperidad para Todos, se fortalece la institucionalidad para atender los temas de género en el país. Puede revisarse en Colombia. Diario Oficial . Ley por la cual se expide el Plan Nacional de Desarrollo, 2010-2014. 16 de Junio de 2011, L 1450/2012, 23 , «Artículo 177. Equidad de género. El Gobierno Nacional adoptará una política pública nacional de Equidad de Género para garantizar los derechos humanos integrales e interdependientes de las mujeres y la igualdad de género, teniendo en cuenta las particularidades que afectan a los grupos de población urbana y rural, afrocolombiana, 
indígena, campesina y Rom. La política desarrollará planes específicos que garanticen los derechos de las mujeres en situación de desplazamiento y el derecho de las mujeres a una vida libre de violencia.

Esta política pública será construida de manera participativa bajo la coordinación de la Alta Consejería para la Equidad de la Mujer (ACPEM), la cual será fortalecida institucional y presupuestalmente para el cumplimiento efectivo de sus responsabilidades y funciones.

Parágrafo. La política pública asegurará el cumplimiento del Estado colombiano de los estándares internacionales y nacionales en materia de Derechos Humanos de las Mujeres con un enfoque multisectorial y transversal».

La revisión de los avances y el replanteamiento de Metas nacionales con respecto al proceso de participación efectiva de la mujer y su proceso de empoderamiento, requieren la definición de indicadores que puedan medir la efectividad de la normatividad y el avance social frente al proceso de equidad con la mujer. En Colombia. Documento Conpes Social Por medio del cual se realiza modificación a Conpes Social 91 del 14 de Junio de 2005: «Metas y Estrategias de Colombia para el logro de los Objetivos de Desarrollo del Milenio - 2015» 28 de Marzo de 2011, C S 140/2011, 12 se ofrecen lineamientos importantes para el logro por parte del país de los ODM para 2015, se presentan algunos indicadores interesantes tales como: «Proporción de escaños ocupados por mujeres en el Congreso de la República, Proporción de mujeres candidatas sobre el total de personas candidatizadas, Proporción de mujeres en los niveles 1 y 2 de las ramas ejecutiva y judicial, a nivel nacional y territorial, el cual actualmente se encuentra en $39 \%$, Brecha en la tasa de participación femenina, indicador que ha venido decreciendo, presenta una línea de base de 34,80 para 1996 , una situación actual de 22,4, se presenta una meta para 2015 de 20, Brecha en la tasa de desempleo, Brecha de ingresos laborales mensuales promedio».

Sin duda éstos indicadores nos permitirán generar un proceso de evaluación de los avances en materia de participación de la mujer en Colombia y su municipalización facilitará las intervenciones que permitan avanzar desde lo local en el empoderamiento de la mujer.

Vale la pena mencionar en éste momento, la generación de líneas de base municipales, para el monitoreo de los avances, pero también la necesidad del surgimiento de investigaciones que indaguen sobre la dinámica de los semilleros de participación, como el caso que nos ocupa en ésta reflexión y que aporten luces sobre el comportamiento de las instancias previas a la participación política de la mujer en los espacios legitimados por la legislación y la sociedad.

La normatividad relacionada ofrece el marco jurídico indispensable para garantizar el derecho a la equidad por parte de las mujeres en Colombia. Visibilizar el tema, es un primer paso en la consecución de la igualdad de derechos, sin embargo se requiere trabajar de manera decidida desde todos los ámbitos, especialmente desde el educativo, para fortalecer la conciencia social y crear un nuevo imaginario colectivo, que permita ver a la mujer como par del hombre en la toma de decisiones, dependiendo de sus capacidades y habilidades, sin ninguna restricción de tipo socio cultural.

En Fondo para el logro de los ODM 2010, se afirma frente a la participación nacional de la mujer: «Si bien el movimiento femenino ha abierto un espacio significativo en términos de incidencia política y de desarrollo de iniciativas legislativas para garantizar el derecho a la participación de las mujeres, aún es incipiente su acceso a instancias de decisión, a pesar de su muy amplia intervención y conformación de redes y organi- 
zaciones sociales de mujeres de muy diversa índole y de su papel crucial en el desarrollo , aún invisibilizado» (p. 82).

La reflexión que nos ocupa pretende ampliar el análisis a lo que se ha considerado como semilleros de participación, en el intento de bajar la legislación al ámbito inmediato, al ámbito local, pues estas dinámicas dan luces con respecto a la evolución de los macro procesos, tratados en la normatividad revisada con antelación.

Específicamente para Boyacá se revisará lo sucedido en los Consejos Municipales de Juventud y los Organismos comunales, con el ánimo de monitorear la incursión de la mujer en éstos espacios de representación social.

\section{Semilleros de participación de la mujer en Boyacá}

A partir de los propósitos universales referenciados anteriormente, se pretende revisar si la dinámica de la participación de la mujer ha venido evolucionando en un Departamento como Boyacá, tradicionalmente con preponderancia masculina. Con éstos antecedentes se esperaría que el cambio ocurra de manera paulatina. En el Departamento se han adelantado algunas investigaciones en la materia por parte de instituciones públicas y privadas. Sin embargo en ésta reflexión, se referencia fundamentalmente la adelantada por la Línea de Infancia, Familia y mujer del Departamento Administrativo de Planeación del Departamento de Boyacá en 2011, de la cual se presentan algunos apartes, para enriquecer la reflexión.

Alba (2011) afirma «Sin duda la mujer, madre y cuidadora del siglo XXI, ha visto como se han acrecentado sus roles y además de tan primordial función en la sociedad, hoy se reconoce también como profesional, como líder comunitaria y social, como empresaria y lucha por el reconocimiento de sus derechos, que en muchos casos son vulnerados e incomprendidos por una sociedad que aún se debate entre los antiguos y los nuevos paradigmas y la evalúa con una visión del siglo XIX, pero espera que esté liderando los procesos del siglo XXI».

Este es otro de los retos que como mujeres debemos superar: éstos mensajes ambivalentes que nos envía la sociedad, y que son producto de un proceso de construcción colectiva, que poco a poco se irá configurando en la imagen de la mujer del siglo XXI, que responda de manera eficiente a todos sus roles, sin olvidar el protagonismo que tiene, el que nos perpetúa como seres humanos: el de ser madre y compañera, constructora de familia (p.4).

Esta tarea encuentra dificultades para ser enfrentada, nos invita a la doble o la triple jornada, nos exige, pero a la vez nos permite demostrarle a la sociedad nuestra capacidad. Muchas mujeres han incursionado ya en los procesos de participación desde los diferentes ciclos vitales; hacen presencia en sus comunidades, desde los Consejos de juventud, desde la estructura comunal, o incipientemente desde el ejercicio de lo político.

A continuación se muestra el comportamiento de la participación de la mujer en estos escenarios, lo cual genera reflexiones frente al cumplimiento del compromiso con el mundo, y frente a si estamos las mujeres preparadas para asumir el reto, si contamos con los suficientes escenarios de participación, si se nos reconoce de acuerdo con nuestras capacidades, habilidades y fortalezas, o si debido a la multiplicidad de roles que asumimos diariamente en la sociedad y en la familia, estamos interesadas en liderar y participar en otros escenarios de decisión.

Las cifras que se presentan a continuación se constituyen en insumos para generar el debate, que sin duda nos ocupará en el próximo milenio. 
Participación de la mujer en Boyacá en los Consejos Municipales de Juventud en 2011

\section{Gráfico 1.}

Participación de la mujer en los Consejos Municipales de Juventud y en el Consejo Departamental de juventud 2011.
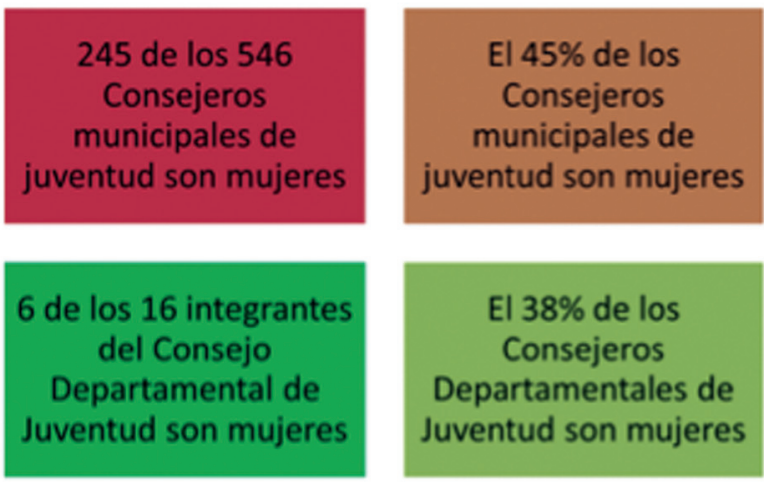

FUENTE: Secretaria de Participación, Gobernación de Boyacá, Proceso Línea de Infancia, Familia y Mujer D.A.P. Boyacá

La participación de los jóvenes ${ }^{8}$ en Boyacá en 2011 realmente es significativa. Se nota un liderazgo importante, que da cuenta de una nueva visión del quehacer de la mujer en la sociedad y seguramente por ser ésta una etapa de la vida en la cual aún no se han asumido responsabilidades de conformación de familia, por ser eminentemente exploratoria, con muchas condiciones deliberantes, se puede observar de acuerdo con las cifras del gráfico 1, con satisfacción, que el $45 \%$ de los Consejeros municipales de juventud son mujeres.

\footnotetext{
${ }^{8}$ En Colombia. Diario Oficial . Ley estatutaria por medio de la cual se expide el estatuto de ciudadanía juvenil y se dictan otras disposiciones. 29 de Abril de 2013, L estatutaria 1622 de 2013, 3. Se encuentra en el «Artículo 5. Definiciones. Para efectos de la presente ley se entenderá como: 1 . Joven. Toda persona entre 14 y

28 años cumplidos en proceso de consolidación de su autonomía intelectual, física, moral, económica, social y cultural que hace parte de una comunidad política y en ese sentido ejerce su ciudadanía. 2. Juventudes. Segmento poblacional construido socioculturalmente y que alude a unas prácticas, relaciones, estéticas y características que se construyen y son atribuidas socialmente. Esta construcción se desarrolla de manera individual y colectiva por esta población, en relación con la sociedad. Es además un momento vital donde se están consolidando las capacidades físicas, intelectuales y morales.... 5. Género. Es el conjunto de características, roles, actitudes, valores y símbolos construidos socialmente que reconoce la diversidad y diferencias entre hombres y mujeres en pleno goce 0 ejercicio de sus derechos y libertades fundamentales, en condiciones de igualdad en las esferas política, económica, social, cultural o en cualquier otra esfera de la vida pública 6. Espacios de participación de las juventudes. Son todas aquellas formas de concertación y acción colectiva que integran un número plural y diverso de procesos y prácticas organizativas de las y los jóvenes en un territorio, y que desarrollan acciones temáticas de articulación y trabajo colectivo con otros actores, dichos espacios deberán ser procesos convocantes, amplios y diversos, y podrán incluir jóvenes no organizados de acuerdo con como espacios de participación entre otros a afrocolombianos, y otros espacios que surjan de las dinámicas de las y los jóvenes»
}

La participación de reduce cuando se asciende en la pirámide decisional al $38 \%$ en los Consejeros Departamentales de juventud.

Puede inferirse que si bien se conquistan nuevos espacios por parte de la mujer, la tendencia de participación se conserva, a mayor rango decisional menor participación de la mujer. Se requiere profundizar en el análisis de la tendencia, para establecer sus causas. Es necesario que se defina una línea de base que permita generar estrategias y acciones para fortalecer la participación de la mujer, así como extender el análisis a los personeros estudiantiles.

\section{Participación de la mujer en Boyacá en los Organismos Comunales elegidos para el periodo 2008-2012}

Vale la pena revisar las cifras para los organismos comunales elegidos en 2008 y hasta 2012. Sin duda en ellos se gesta la participación, por cuanto éste corresponde al nivel más próximo a las necesidades de la comunidad, si bien no cuenta con recursos económicos para ofrecer soluciones, si se convierte en una alternativa de gestión para encontrarlas. ${ }^{9}$

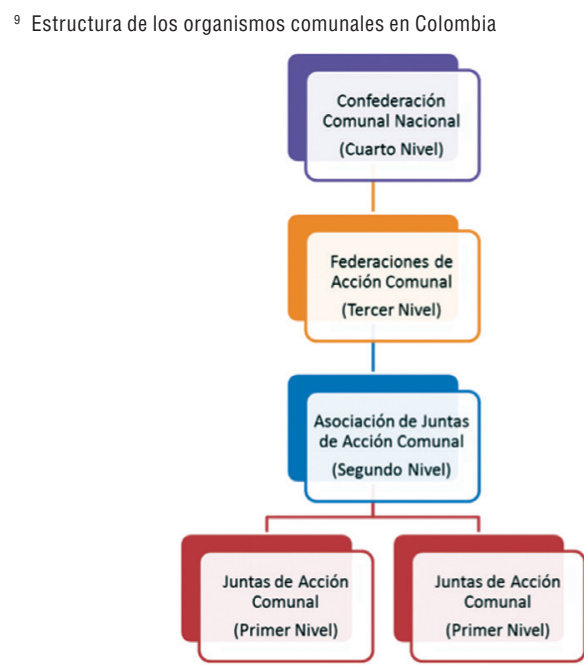

FUENTE: Sub-dirección de asuntos comunales, Instituto Distrital de Participación y Acción Comunal Bogotá D.C., proceso Línea de Infancia, Familia y Mujer D.A.P Boyacá 
Resulta pertinente retomar y revisar algunas cifras del estudio realizado por el Departamento Administrativo de Planeación consignadas en el gráfico 2.

En el primer nivel de la estructura de las Juntas de Acción Comunal ${ }^{10}$, se contaba para 2011 con cerca de 20.000 miembros y 7000 mujeres, distribuidas en los diferente órganos consultivos, siendo el de mayor relevancia el de administración ${ }^{11}$. Allí se cuenta con un $35 \%$ de mujeres participando, cifra representativa de la dinámica que se gesta en los movimientos comunales.

Sin embargo cabe anotar que del total de cargos dentro de ésta estructura, el mayor nivel de participación de la mujer se encuentra como secretaria. Puede interpretarse éste resultado en dos sentidos: teniendo en cuenta la capacidad organizativa de la mujer y su condición de relatora innata, o por un proceso de marginación hacia los cargos de decisión.

De los 2609 nombrados en el cargo, el $71 \%$ son mujeres, ocupando el ranking más alto el Municipio de Busbanzá con 104, seguido de Cuitiva con 101, Guacamayas con 71, Oicatá con 62 y Santana con 52, Chíquiza es el municipio en donde hay un menor grado de participación en éste cargo con 2 mujeres. Cabe anotar que la participación en éste municipio es muy baja en todos los órganos consultivos.

El 33\% de los 2622 miembros que aparecen registrados como tesoreros de las juntas son mujeres, siendo el municipio con el ranking mas alto Cerinza con 62, seguido de Santana con 58, Busbanzá con 34 y Chivatá con 33. No hay ninguna participación en éste sentido en los municipios de Chiquiza, Corrales, Oicatá y San Eduardo.

\footnotetext{
${ }^{10}$ Análisis realizado para 121 municipios que reportaron datos a la Secretaria de participación y Democracia y que fueron involucrados en su sistema de información.

Órgano de Administración de las Juntas de Acción Comunal: hacen parte de él el Presidente (a), vicepresidente(a), Tesorero (a) y Secretario (a): cuyo perfil está orientado a la gestión y la organización comunitaria, así como a dar cuenta de los recursos que eventualmente sean asignados para sus comunidades, de acuerdo con las necesidades que hayan sido detectadas.
}

La proporción sigue disminuyendo a medida que se asciende en la jerarquía del cargo. El $20 \%$ de los 2517 miembros que aparecen registrados como Vice - presidentes son mujeres. Ocupando el ranking mas alto Chivatá con 119 (Vale la pena mencionar que éste municipio cuenta actualmente con Alcaldesa), Pisba con 110, Cerinza con 87, Somondoco con 74 y Chitaraque con 67. En los municipios de Sora, Toca y Viracachá se registra la menor participación de la mujer para el cargo.

Gráfico 2. Participación de la mujer en Las Juntas de Acción Comunal en Boyacá periodo 2008-2012 (Alba, 2011:10).

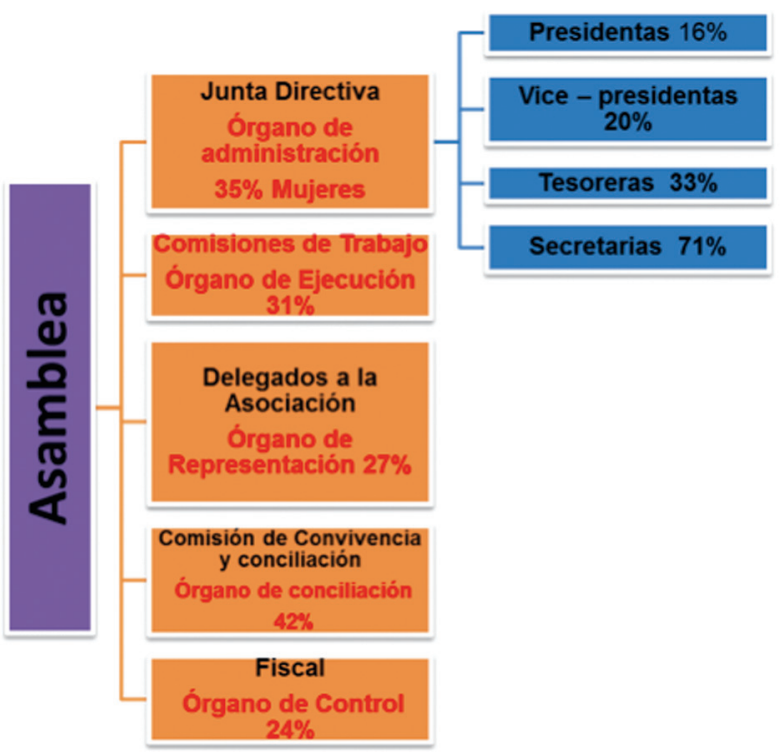

FUENTE: Secretaria de Participación, Gobernación de Boyacá, Proceso Línea de Infancia, Familia y Mujer D.A.P. Boyacá

Para el caso de Presidentes, de los 2584 registrados, el $16 \%$ son mujeres, siendo Oicatá el municipio con el ranking mas alto alcanzando 28 Presidentas, seguido de Almeida con 24, Guateque con 20, Tibasosa con 19 y Cerinza con 14. Para el caso se amplia el número de municipios (20) que no cuentan con ninguna Presidenta, vale la pena mencionar a Chíquiza, Corrales, San Eduardo, Busbanzá y Arcabuco. 
En los gráficos 3, 4 y 5 se puede observar la tendencia de la participación de la mujer en los diferentes niveles de la estructura de los organismos comunales de Boyacá. A medida que se asciende el en nivel de jerarquía disminuye la participación de la mujer. Se generan entonces interrogantes frente a la situación: ¿le falta protagonismo a la mujer debido a su escasa formación?, ¿encuentra cuellos de botella culturales que no le permiten descollar en los niveles directivos mas altos? ( Como es el caso de Chíquiza municipio con costumbres ancestrales de corte machista que aún permanecen y que se reflejan en la baja participación de la mujer en las juntas de Acción comunal, entre otros aspectos relevantes ), ¿no existe interés por parte de la mujer para ocupar éstos cargos debido a su posición en la familia como cuidadora?.

Se requiere profundizar en éstos aspectos de la investigación para responder los interrogantes planteados y generar, de requerirse, los espacios y las oportunidades que consoliden el proceso de participación de la mujer.

Gráfico 3. Participación de la mujer en el Órgano de Administración de las Juntas de Acción Comunal de Boyacá 2008-2012

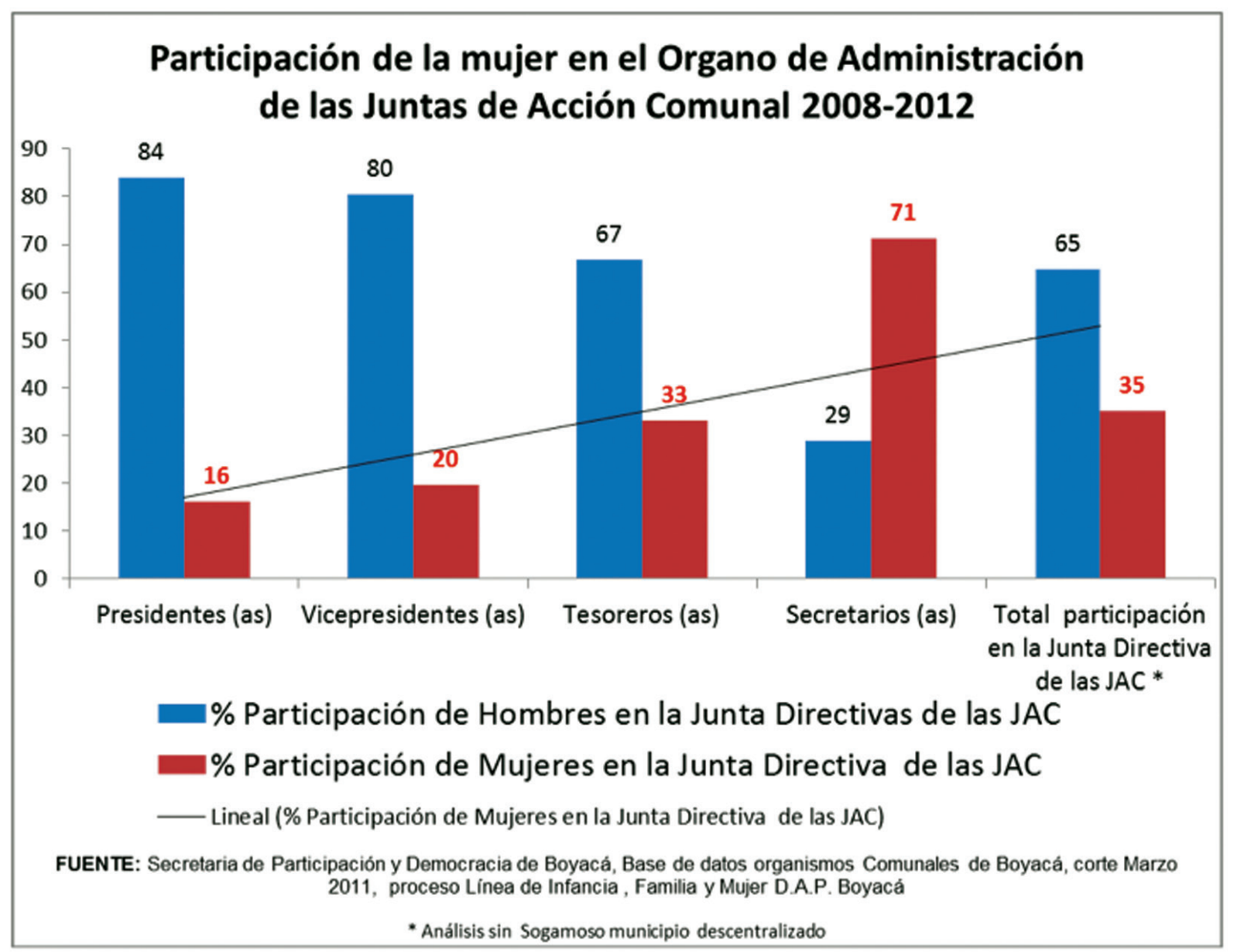

FUENTE: Secretaria de Participación, Gobernación de Boyacá, Proceso Línea de Infancia, Familia y Mujer D.A.P. Boyacá 
Gráfico 5. Participación de la mujer por órganos constitutivos en las Asociaciones Municipales de Juntas de Acción Comunal Boyacá 2008
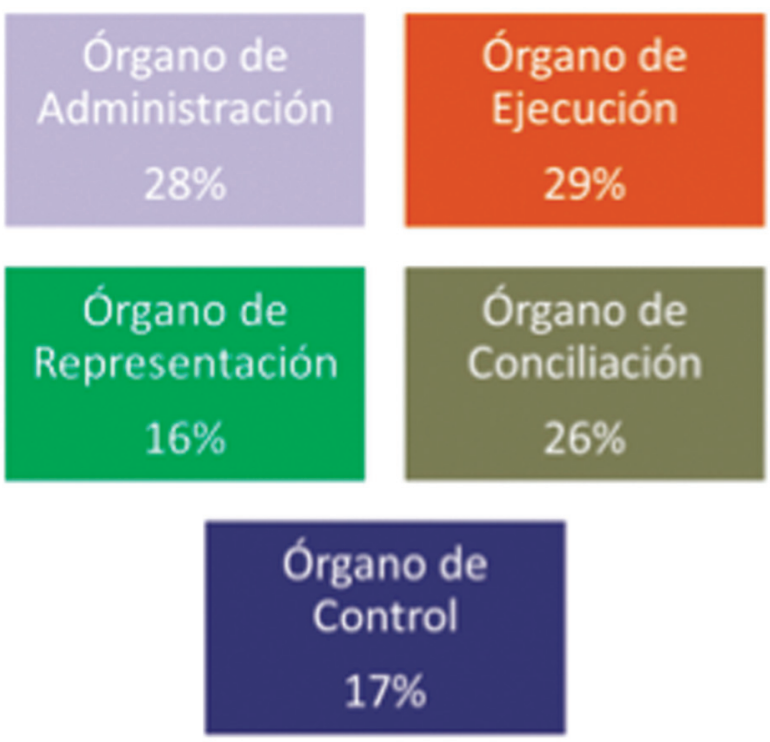

FUENTE: Secretaria de Participación, Gobernación de Boyacá, Proceso Línea de Infancia, Familia y Mujer D.A.P. Boyacá

Mientras la proporción de la participación de la mujer en las juntas Directivas del primer nivel en el órgano de administración era del $35 \%$, en el mismo órgano del segundo nivel, en las Asociaciones Municipales es del $28 \%$ y de $0 \%$ en la Federación de Juntas de Boyacá, lo mismo sucede en el órgano de ejecución que pasa de 31 a 29\%, y al 10\% en la Federación, en el de representación se pasa de un $27 \%$ a un $16 \%$ como delegados a la Federación, que se convierte en $0 \%$ para la Confederación.

En cuanto al órgano de conciliación la proporción pasa de un $42 \%$ en las Juntas de Acción comunal a un $26 \%$ en la Asociación Municipal de Juntas y a un $0 \%$ en la Federación. En cuanto al órgano de control se pasa de un $24 \%$ a un $17 \%$ en la Asociación de municipios y a un $0 \%$ en la Federación (Alba, 2011:18).
Gráfico 6. Participación de la mujer en la Federación de Juntas de Acción Comunal Boyacá 20082012 (Alba, 2011:18).

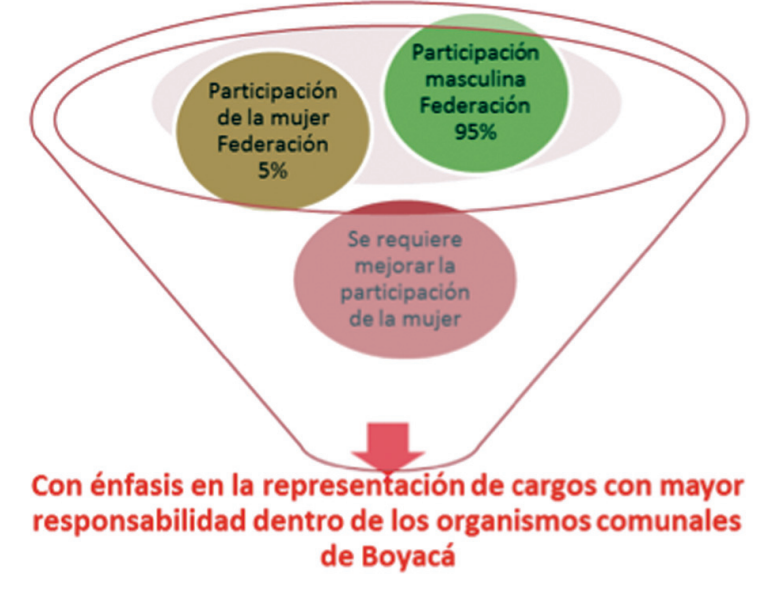

FUENTE: Secretaria de Participación, Gobernación de Boyacá, Proceso Línea de Infancia, Familia y Mujer D.A.P. Boyacá

Después de revisadas las cifras puede concluirse que existe una inquietud importante de la mujer por participar en los escenarios comunales, pero además está latente una responsabilidad de parte de los actores académicos y es la de formar a éste importante sector del Departamento, otorgándole las condiciones necesarias para que puedan aprovecharse de manera efectiva las oportunidades que la sociedad del siglo XXI ha generado.

\section{Conclusiones}

- Se presentan avances significativos en el país frente a la generación del marco normativo que permite consolidar los procesos de participación de la mujer en los escenarios de decisión.

- Se cuenta con una base de indicadores de monitoreo que permiten visibilizar los avances de política en materia de participación de la mujer y que sirven de fundamento para la rendición de cuentas frente al Objetivo tres de Desarrollo del Milenio: Promover la igualdad entre géneros y la autonomía de la mujer. 
- Se requiere la generación de líneas de base municipales que monitoreen los semilleros de liderazgo, para visibilizar la dinámica de la participación de la mujer.

- En Boyacá se muestran avances significativos en la participación de la mujer en el ciclo vital de juventud, pues en los Consejos Municipales de Juventud, se nota una dinámica interesante, que alcanza el $45 \%$ de los escaños disponibles en 2011.

- En general se observa que la participación de la mujer a medida que se avanza en la jerarquía del nivel decisorio se reduce significativamente, tanto en los concejos Municipales de Juventud como en los Organismos comunales.

- Se requiere profundizar en las investigaciones para dar respuestas a temas tan sensibles tales como:

a. ¿Están las mujeres preparadas para asumir el reto de la participación y el liderazgo o les falta protagonismo debido a su escasa formación académica?

b. ¿Está la sociedad preparada para asumir de manera adecuada el liderazgo de la mujer?

c. ¿La mujer cuenta con los suficientes escenarios de participación, o apenas nos encontramos en una etapa de desarrollo normativo, que la sociedad no ha logrado asumir?

d. ¿A las mujeres se les reconoce de acuerdo con sus capacidades, habilidades y fortalezas ? o por el contrario nos encontramos en el transito de un proceso de reconocimiento. e. Debido a la multiplicidad de roles que asume diariamente la mujer en la sociedad y en la familia, ella está interesada en liderar y participar en otros escenarios de decisión? O por el contrario no existe interés de su parte para ocupar éstos cargos debido a su posición en la familia como cuidadora? Existe la posibilidad de que el derecho vaya por un lado y el deseo por el otro.

f. ¿La mujer encuentra cuellos de botella culturales que no le permiten descollar en los niveles directivos mas altos? ( Como es el caso de Chíquiza, municipio con costumbres ancestrales de corte machista que aún permanecen y que se reflejan, para el caso objeto de análisis, en la baja participación de la mujer en las juntas de Acción comunal)

g. ¿El modelo actual de familia puede ajustarse sin mayores traumatismos a la creciente participación de la mujer en los escenarios de decisión?

El tema de la participación es complejo, requiere de formación, generación de oportunidades y posibilidades reales para ejercer los derechos reconocidos, pero también invita a la mujer a asumir nuevos roles, que deben ser compartidos con la estructura familiar en evolución, con la participación en el sistema de producción, con el desarrollo profesional y del ser. Estos son solo algunos de los retos del siglo XXI para la sociedad, el estado, la academia y la mujer, la discusión está planteada y solamente nuestra capacidad para elegir adecuadamente un proyecto de vida que responda a nuestras inquietudes y materialice nuestros derechos, nos entregarán las respuestas que la sociedad del conocimiento y del reconocimiento nos platean. 


\section{Referencias}

Alba Sánchez Doris Amalia (2011). Reflexiones de Política Social para Boyacá, Línea de Infancia, Familia y Mujer, Tunja: Departamento Administrativo de Planeación de Boyacá.

Alba Sánchez Doris Amalia, (2011). Investigación Participación de la Mujer en Boyacá, Línea de Infancia, Familia y Mujer, Tunja: Departamento Administrativo de Planeación de Boyacá.

Barraza M. Cecilia, (2007). Objetivos de Desarrollo del Milenio. Cartilla de Buenas Prácticas para «Promover la igualdad entre los sexos y la autonomía de la mujer» en el ámbito local. PNUD- Colombia. Noviembre de 2007.

Colombia. Diario Oficial . Ley estatutaria por la cual se reglamenta la adecuada y efectiva participación de la mujer en los niveles decisorios de las diferentes ramas y órganos del poder público, de conformidad con los artículos 13, 40 y 43 de la Constitución Nacional y se dictan otras disposiciones. 31 de mayo de 2000. L estatutaria $581 / 2000,1-2$.

Colombia. Presidencia de la República. Decreto por el cual se crean unas Altas Consejerías en el Departamento Administrativo de la Presidencia de la República. 17 de septiembre de 2010. D 3445/2010, 7.

Colombia. Diario Oficial. (2011). Por la cual se modifica y adiciona la Ley 5 de 1992, se crea la Comisión Legal para la Equidad de la Mujer del Congreso de la República de Colombia y se dictan otras disposiciones. 6 de Enero de 2011, L 1434/2011, 1.

Colombia. (2011). Documento Conpes Social Por medio del cual se realiza modificación a Conpes Social 91 del 14 de Junio de 2005: «Metas y Estrategias de Colombia para el logro de los Objetivos de Desarrollo del Milenio - 2015» 28 de Marzo de 2011, C S 140/2011, 12
Colombia. (2011). Diario Oficial. Ley por la cual se expide el Plan Nacional de Desarrollo, 2010-2014. 16 de Junio de 2011, L 1450/2011, 23

Colombia. (2011). Diario Oficia . Ley estatutaria por medio de la cual se adoptan Reglas de organización y funcionamiento de los partidos y movimientos políticos, de los procesos electorales y se dictan otras disposiciones. 14 de Julio de 2011, L estatutaria 1475/2011, 12.

Colombia. (2013). Diario Oficial. Ley estatutaria por medio de la cual se expide el estatuto de ciudadanía juvenil y se dictan otras disposiciones. 29 de Abril de 2013, L estatutaria 1622 de 2013.

Fondo para el Logro de los ODM. (2010). Cátedras del Milenio. Objetivos de Desarrollo del Milenio. Bogotá: Editorial Nuevas Ediciones S.A.

PNUD. (2012). Estado de avance de los Objetivos de Desarrollo del Milenio, Boyacá .

PNUD, (2003). Informe sobre Desarrollo Humano.

Mendez Alvarez, Carlos Eduardo. (1998). Metodología. Guía para elaborar diseños de investigación en ciencias económicas, contables y administrativas. Bogotá: Editorial McGraw- Hill, Segunda Edición.

Tamayo y Tamayo, Mario, (2002). El proceso de la investigación científica. Incluye evaluación y administración de proyectos de investigación, México: Editorial Limusa.

Sánchez Torres, Carlos Ariel. (2012). Nuestra Huella, Registraduría Nacional del Estado Civil. Revista Electrónica Mensual. Edición Nro. 70. Año VI. Diciembre de 2012. 